\title{
What is the role of PSMA PET in salvage prostatectomy?
}

\author{
Goonewardene $\mathrm{SS}^{1 *}$ and Cahill $\mathrm{D}^{2}$ \\ ${ }^{1}$ Guys and St Thomas Hospitals, London, UK \\ ${ }^{2}$ The Royal Marsden Hospital, London, UK
}

\section{Letter to Editor}

Salvage surgery for prostate cancer has advanced significantly with the advent of robotics. Detection of recurrence is a major challenge for imaging [1]. There is an ongoing need for an accurate imaging for investigation of recurrent disease and salvage therapy in prostate cancer [2]. However, with regards to diagnostics in salvage robotic prostatectomy, what else can we use to improve outcomes?

PSMA PET scanning is a new method of nuclear imaging which can be used in salvage therapy. Prostate-specific membrane antigen [PSMA] is a type II transmembrane protein with high expression in prostate carcinoma cells [3]. This allows for cancer cell targeting [1]. A number of PSMA-targeting molecules are available [4]. These have been used with similar success compared to choline PET [1]. The relevance of this is increasing continually increasing especially in the field of salvage therapy [5].

Mittal et al. demonstrated that gallium labelled PSMA PET/CT is better to detect recurrence in patients with prostate cancer [1]. A higher number of lesions viewed and improved contrast uptake is achieved as compared to $18 \mathrm{~F}$-choline PET/CT [1]. This demonstrates high tumourto background ratios and good detection rates as a result [6].

Recently, a PSMA-targeted theranostic concept has been successfully implemented by [5]. Giesel et al. used this technique as part of assessment for salvage therapy [7]. Gallium labelled PSMA ligand PET/CT had a dramatic impact on salvage therapy management [7]. In $50.8 \%$ of the cases the salvage therapy was changed as a result of imaging. This demonstrates the presented imaging technique of Gallium PSMA PET/CT could be a key technology for individualized salvage therapy management in prostate cancer. This again highlights the ability of this technique to detect recurrence.

Freitag et al. evaluated the reproducibility of the combination of hybrid PET/MRI and the Gallium labelled PSMA-11 tracer in depicting lymph node and bone metastases of prostate cancer in comparison with that of PET/CT [8]. This again demonstrated nodal and osseous metastases are accurately assessed by hybrid PET/MRI [8]. This demonstrates PSMA PET can be successfully used to detect lymph node metastases and bony metastases as part of salvage management.

This was further confirmed by Lutje et al. [9]. Serum PSA levels in recurrence linearly correlate with uptake of the 68Ga-PSMA ligand in terms of standardised uptake values [9]. Strongest correlation was observed for bone metastases [9]. In addition, the serum PSA level correlated with the number of tumour lesions detected with $68 \mathrm{Ga}$ PSMA-PET/CT [9]. This can help with selection of patients for PSMA PET [9] and specific salvage therapies.

Demirci et al., evaluated the diagnostic value of PSMA PET/CT in recurrence with low PSA levels [10]. This paper confirms PSMA PET/ CT has a high sensitivity [\%79.3] within the patients who has PSA levels between $0.2-5 \mathrm{ng} / \mathrm{ml}[10]$. Additionally PSMA PET/CT can be used in patients with very low $[<0.2 \mathrm{ng} / \mathrm{ml}]$ but increasing PSA levels, which in many cases may influence the further clinical management [10]. This quite clearly highlights the PSA levels at which PSMA PET can be useful in salvage therapy diagnostics. Most importantly, it reveals a high number of positive findings in the clinically important range of low PSA values $[<0.5 \mathrm{ng} / \mathrm{mL}]$, which in many cases can substantially influence the further clinical management [11].

When PSA was below $0.5 \mathrm{ng} / \mathrm{mL}$, the detection rate was $50 \%$. When PSA was $0.5-2.0 \mathrm{ng} / \mathrm{mL}$, the detection rate was $69 \%$ and when PSA was above 2.0 , the detection rate was $86 \%$ [12].

There were 42 [89\%] positive [PSA 0.48-56] and 5 [11\%] negative studies [PSA 0.21-1.9] [13]. All patients with PSA $>2$ had positive studies [33], compared to 9/14 [64\%] patients with PSA $<2$ [13].

The first evaluation of pelvic extended lymph node dissection [pLND] in oligometastatic prostate cancer PSMA PET was conducted and highlighted a number of points [14]. Retrospective analysis of 35 patients demonstrated after pLND, the immediate biochemical relapse free rates [PSA never measured less than $0.2 \mathrm{ng} / \mathrm{ml}$ ] was $18 \%$ [14]. This represents the first study of pLND in the setting of nodal oligometastatic PCA detected by PSMA PET [14]. This demonstrates the need to improve the accuracy in staging and detection of nodal micrometastases in salvage therapy [14].

Both primary and metastatic prostate carcinomas and PSMA PET scanning have been evaluated. This demonstrates rapid and early uptake of Galiium-68 PSMA within minutes of injection and markedly higher uptake rates compared to normal tissues [15]. This demonstrated early imaging of PSMA may offer improved depiction of disease burden with less confounding bladder or ureteric activity [15]. Additionally what was also demonstrated was uptake in prostate cancer recurrence increases significantly over time [16]. Two phase scanning may help to differentiate between benign lesions and PSMA-expressing prostate cancer-relapses or -metastases [16]. The overall conclusion here was early imaging lead to better outcomes.

With the advent of modern fusion biopsy systems, suspicious areas more accurately assed and increased detection rates are reported [17].

Correspondence to: Guys and St Thomas Hospitals, London, UK, E-mail: ssg7727@yahoo.co.uk

Key words: PSMA PET, prostatectomy, robotics, salvage therapy

Received: May 10, 2016; Accepted: June 21, 2016; Published: June 24, 2016 
In this initial analysis, PSMA-PET/MRI in the combination with a newly developed fusion biopsy system proved as valuable tool for the detection of recurrent prostate cancer after prior negative prostate biopsy [18].

In conclusion, PSMA PET has a significant role to play in salvage therapy, in detection of localised, locally advanced and metastatic disease as well as treatment.

\section{References}

1. Mittal BR, Shukla J, Parghane RV, Phulsunga RK, Vatsa R, Bhusari P, et al. (2015) Ga68 labelled PSMA ligand based PET/CT scan for the detection of recurrence in prostate cancer patient and comparison with F-18 choline-based PET/CT scan in a tertiary care center. European Journal of Nuclear Medicine and Molecular Imaging 1: S384-S5.

2. Voo S, Van Kroonenburgh A, Mottaghy FM (2015) Ga-68 labelled PSMA in prostate cancer: Its diagnostic value in patients with biochemical recurrence of prostate cancer. European Journal of Nuclear Medicine and Molecular Imaging 1: S34.

3. Komek H, Elboga U, Can C, Basibuyuk M, Celen Y (2015) Biodistribution of Ga-68 labelled PSMA ligand in normal tissues with PET/CT imaging. European Journal of Nuclear Medicine and Molecular Imaging 1: S406.

4. Haberkorn U, Babich JB, Kopka K, Eder M, Eisenhut M (2015) PSMA ligands for diagnosis and therapy. Radiotherapy and Oncology 115: S293.

5. Schottelius M, Wirtz M, Eiber M, Maurer T, Wester HJ. (2015) [ $\left.{ }^{111} \mathrm{In}\right]$ PSMA-I\&T: expanding the spectrum of PSMA-I\&T applications towards SPECT and radioguided surgery. EJNMMI Research 5: 1-5.

6. Artigas C, Wimana Z, Ghanem G, Vanderlinden B, Flamen P (2015) First clinical experience using a cold kit based 68Ga-PSMA PET/CT for recurrence detection of prostate cancer. European Journal of Nuclear Medicine and Molecular Imaging 1: S674-S5.

7. Giesel F, Fiedler H, Stefanova M, Kratochwil C, Afshar-Oromieh A, et al. (2015) 68Ga-PSMA11-PET/CT-a new technique with high potential for the radiotherapeutic management of prostate cancer patients. European Journal of Nuclear Medicine and Molecular Imaging 1: S174-S5.

8. Freitag MT, Radtke JP, Hadaschik BA, Kopp-Schneider A, Eder M, et al. (2016) Comparison of hybrid ${ }^{68} \mathrm{Ga}$-PSMA PET/MRI and ${ }^{68} \mathrm{Ga}-\mathrm{PSMA}$ PET/CT in the evaluation of lymph node and bone metastases of prostate cancer. European Journal of Nuclear Medicine and Molecular Imaging 43: 70-83.
9. Lutje S, Gomez B, Heskamp S, Reichel R, Bockisch A, et al. (2015) Correlation of serum PSA levels with 68Ga-PSMA tumor SUV in patients with primary and metastatic prostate cancer. European Journal of Nuclear Medicine and Molecular Imaging 1: S671.

10. Demirci E, Nematyazar J, Akyel R, Razavi B, Aygun A, et al. (2015) Diagnostic value of (68)Ga - PSMA PET/CT in biochemical recurrence of prostate carcinoma with low PSA levels. European Journal of Nuclear Medicine and Molecular Imaging 1: S224.

11. Maurer T, Beer AJ, Souvatzoglou M, Holzapfel K, Kubler H, et al. (2014) 68Galliumlabelled ligand of prostate-specific membrane antigen (PSMA) for the evaluation of recurrent prostate cancer using PET/CT and PET/MR imaging. European Urology, Supplements 13: e726.

12. Morigi JJ, Stricker PD, van Leeuwen PJ, Tang R, Ho B, et al.(2015) Prospective Comparison of 18F-Fluoromethylcholine Versus 68Ga-PSMA PET/CT in Prostate Cancer Patients Who Have Rising PSA After Curative Treatment and Are Being Considered for Targeted Therapy. Journal of nuclear medicine : official publication, Society of Nuclear Medicine 56: 1185-1190.

13. Zhou W, Schembri G, Aslani A, Bailey D, Kneebone A, et al. (2015) Correlating the extent of $\left[{ }^{68} \mathrm{Ga}\right]$-PSMA PET positive disease with pre-scan PSA in prostate cancer Journal of Nuclear Medicine Conference: Society of Nuclear Medicine and Molecular Imaging Annual Meeting.

14. Hijazi S, Meller B, Leitsmann C, Strauss A, Meller J, Ritter CO, et al. (2015) Pelvic lymph node dissection for nodal oligometastatic prostate cancer detected by ${ }^{68} \mathrm{Ga}$ PSMA-positron emission tomography/computerized tomography. Prostate 75: $1934-$ 1940.

15. Hsiao E, Schembri GP, Bailey DL, Bailey EA, Roach PJ (2015) Pelvic dynamic imaging with pixel-based analysis of Ga-68 PSMA PET/CT. European Journal of Nuclear Medicine and Molecular Imaging 1: S34-S5.

16. Sahlmann CO, Meller B, Bouter C, Ritter CO, Ströbel P, et al. (2016) Biphasic (68)GaPSMA-HBED-CC-PET/CT in patients with recurrent and high-risk prostate carcinoma. Eur J Nucl Med Mol Imaging 43: 898-905. [Crossref]

17. Storz E, Shah A, Zettinig O, Eiber M, Wester HJ, et al. (2015) PSMA-PET/MRI-guided transrectal fusion biopsy for the detection of prostate cancer. European Urology, Supplements 14: e217.

18. Frisch B, Storz E, Zettinig O, Shah A, Kubler H, et al. (2015) PET/MRI/TRUS image fusion guided prostate biopsy: Development of a research platform and initial clinical results. Journal of Nuclear Medicine Conference: Society of Nuclear Medicine and Molecular Imaging Annual Meeting, SNMMI.

Copyright: (C)2016 Goonewardene SS. This is an open-access article distributed under the terms of the Creative Commons Attribution License, which permits unrestricted use, distribution, and reproduction in any medium, provided the original author and source are credited. 